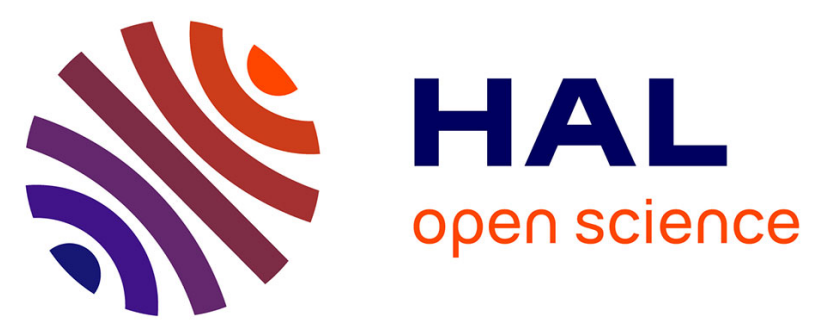

\title{
MATERIAL CHARACTERIZATION BY AN INNOVATIVE BIAXIAL SHEAR EXPERIMENT AT VERY LARGE STRAINS AND AT VERY HIGH STRAIN RATES
}

C. Albertini, L. Griffiths, M. Montagnani, A. Rodis, P. Mariotti, A. Paluffi, G. Pazienza

\section{To cite this version:}

C. Albertini, L. Griffiths, M. Montagnani, A. Rodis, P. Mariotti, et al.. MATERIAL CHARACTERIZATION BY AN INNOVATIVE BIAXIAL SHEAR EXPERIMENT AT VERY LARGE STRAINS AND AT VERY HIGH STRAIN RATES. Journal de Physique IV Proceedings, 1991, 01 (C3), pp.C3435-C3-440. 10.1051/jp4:1991361 . jpa-00250505

\section{HAL Id: jpa-00250505 https://hal.science/jpa-00250505}

Submitted on 1 Jan 1991

HAL is a multi-disciplinary open access archive for the deposit and dissemination of scientific research documents, whether they are published or not. The documents may come from teaching and research institutions in France or abroad, or from public or private research centers.
L'archive ouverte pluridisciplinaire HAL, est destinée au dépôt et à la diffusion de documents scientifiques de niveau recherche, publiés ou non, émanant des établissements d'enseignement et de recherche français ou étrangers, des laboratoires publics ou privés. 
JOURNAL DE PHYSIQUE IV

Colloque C3, suppl. au Journal de Physique III, Vol. 1, octobre 1991

\title{
MATERIAL CHARACTERIZATION BY AN INNOVATIVE BIAXIAL SHEAR EXPERIMENT AT VERY LARGE STRAINS AND AT VERY HIGH STRAIN RATES
}

\author{
C. ALBERTINI*, L.J. GRIFFITHS*, M. MONTAGNANI*, A. RODIS* \\ P. MARIOTTI**, A. PALUFFI* and G. PAZIENZA* \\ "Commission of the European Communities, Joint Research \\ Centre, Ispra Site, I-21020 Ispra (Va), Italy \\ ** ото Melara, P.O. Box 71, I-54011 Aulla (Massa Carrara), \\ Italy
}

Résumé - Afin de déterminer les propriétés des matériaux dans les conditions de coupe des métaux, d'étirage ou de pénétration, une expérimentation en cisaillement biaxial a été réalisée pour de grandes déformations $(\sim 4)$ et très grandes vitesses de déformation $\left(0,4 \times 10^{5} \mathrm{~s}^{-1}\right)$ : une éprouvette spéciale est utilisée, présentant une partie utile annulaire de faible épaisseur $(0,25 \mathrm{~mm})$. Le chargement est effectué à l'aide d'une barre d'Hopkinson en tension-torsion qui peut également être utilisée à faibles et moyennes vitesses de déformation. Une technique électro-optique particulière a été développée pour mesurer directement les déplacements axiaux et angulaires, ainsi que les contraintes de cisaillement. Les courbes contraintedéformation équivalentes obtenues pour l'acier AISI 316 sont comparées aux courbes de traction.

ABSTRACT

In order to measure the material properties in the conditions of metal cutting, wire drawing and missile penetration, a biaxial shear experiment has been performed at very large. strains $(\sim 4)$ and at very high strain rate $\left(0.4 \times 10^{5} \mathrm{~s}^{-1}\right)$ using a special specimen having a thin $(0.25 \mathrm{~mm})$ ring-shaped gauge part. The loading device is a tension-torsion Hopkinson bar apparatus which can be used also at low and medium strain rate. A special electroptical technique has been developed for the direct measurement on the specimen of the axial and angular displacements and of the shear stresses. Equivalent stress strain curves from shear tests of AISI 316 have been compared with tension curves.

\section{INTRODUCTION}

Most of the viscoplastic constitutive models of metals used in numerical calculation codes are generalized to multi-axial loading and to a particular deformation mode by means of classical yielding criteria (e.g. Von Mises, Tresca). Despite their extensive use, such yielding criteria have very seldom been verified at large strains and at very high strain rates. The pressure-shear experiment, which involves the high-speed skewed impact of flyer plates / $/$, allows very high strain rates $\left(\sim 10^{5} \mathrm{~s}^{-1}\right)$ but is limited to small strains. Furthermore, recent results from different laboratories /2/ show that at large strains it is not possible to obtain a unique equivalent flow curve for a metal subjected to a tension, compression or shear test. These latter results raise uncertainty about the meaningful interpretation of biaxial experiments with mixed deformation modes such as the skewed impact flyer plates experiment and the more usual tension-torsion experiment on thinwalled tubes. Therefore, we have proposed and describe here a biaxial shear experiment which has the aim of testing at very large strains and at very high strain rates with the same deformation mode in two orthogonal loading directions.

The constitutive behaviour obtained from the results of the new experiment should 
be very relevant to the prediction of metal cutting processes, high-speed wire drawing and impact penetration processes - all of which are characterized by complex shear stresses, large strains and very high strain rates.

\section{THE BIAXIAL SHEAR SPECIMEN}

The biaxial shear specimen, represented in Fig.1, has a gauge part consisting of a thin circular ring of rectangular cross-section formed between two cylinders of slightly different diameter by machining from a solid bar of material. The ring has an axial length $h$, a thickness a (gauge length) and corners of external radius $r$. Parametric elasto-plastic numerical calculations $/ 3,4 /$, together with interrupted experiments and microscopical observations $/ 4 /$, were used to fix the optimum size of the gauge part in order to minimize undesired stress components and stress concentrations. The optimum size was found by fixing the ratios $a / h=0.5$ and $r / h=0.2$ (Fig.1).

The actual shear specimen was constructed with a short gauge length a $=0.25 \mathrm{~mm}$, which allows a homogeneous stress distribution along the gauge length even in the case of very high strain rates.

In spite of the shortness of the gauge length, the specimen remains representative of the mechanical properties of metals having a grain size up to 50 micron typical of a large class of industrial metal alloys.

The gauge part of the specimen can be loaded in biaxial shear stress by applying an axial load and a torque simultaneously to the cylindrical ends of the specimen. Due to its resemblance to a small calice glass, the specimen has been given the Italian name 'bicchierino'.

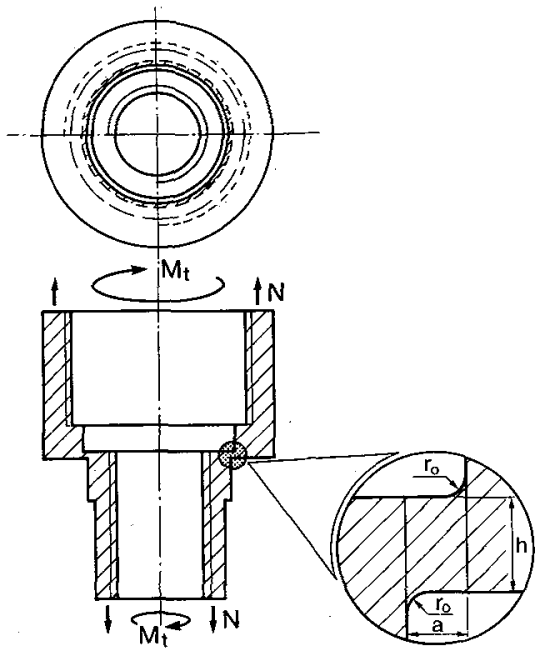

Fig.1: Double shear specimen "bicchierino".

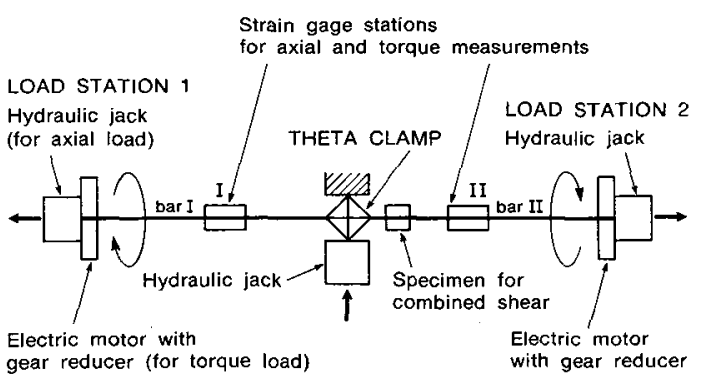

Fig.2: Tension-torsion loading apparatus.

\section{DESCRIPTION OF THE TENSION-TORSION LOADING APPARATUS}

The loading apparatus consists of two bars of about equal length (Fig.2), each connected at one end to the specimen and at the other end both to a hydraulic actuator for tensioning the bar and to an electric motor with gear reducer to generate torque in the bar. The apparatus can work as a tension-torsion loading apparatus for strain rates ranging between $10^{-3}$ and $10^{2} \mathrm{~s}^{-1}$ by applying the tension and the torque directly to the specimen through the bars. The duration of the test is very long in comparison to the time of propagation of stress waves in the bars and so, in this case, the test can be carried out at quasi-static and medium strain rate. The apparatus works as a torsion-tension Hopkinson bar system (so that wave propagation is taken into account) when one of the two bars, e.g. bar. I in Fig.2, is 
constrained near the specimen by means of a special device named a theta clamp (Fig.3). The clamp constraints the bar over a short length of about $25 \mathrm{~mm}$ by the friction of a split collar 'pinched' by a hydraulic actuator operating perpendicularly to the bar axis. The necessary load from the actuator is transmitted to the collar through four metal rafters (Fig.3) set in a parallelogram together with a 'brittle bridge'. Further loading of the actuator breaks the bridge, releases the bar at the clamp, and allows an axial tensile pulse and a torsional pulse to propagate down the bar to load the specimen. These pulses travel at different speeds so that when the 'theta clamp' is positioned far from the specimen, it is subjected to the two modes of loading consecutively. However, if the clamp is placed sufficiently close to the specimen, the pulses arrive together and virtually simultaneous loading is achieved.

More complex strain-rate and strain histories can be obtained by superposing the direct slow action on the specimen of one of the bars and the fast action of the tension and torsional pulses generated by the other bar.

A possible complex strain and strain-rate history is represented in Fig.4.

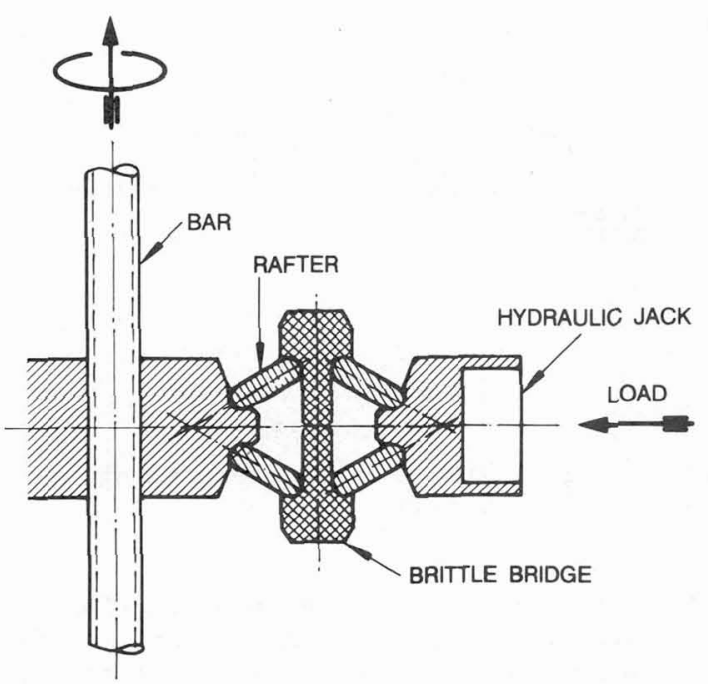

Fig. 3: "Theta" clamp.
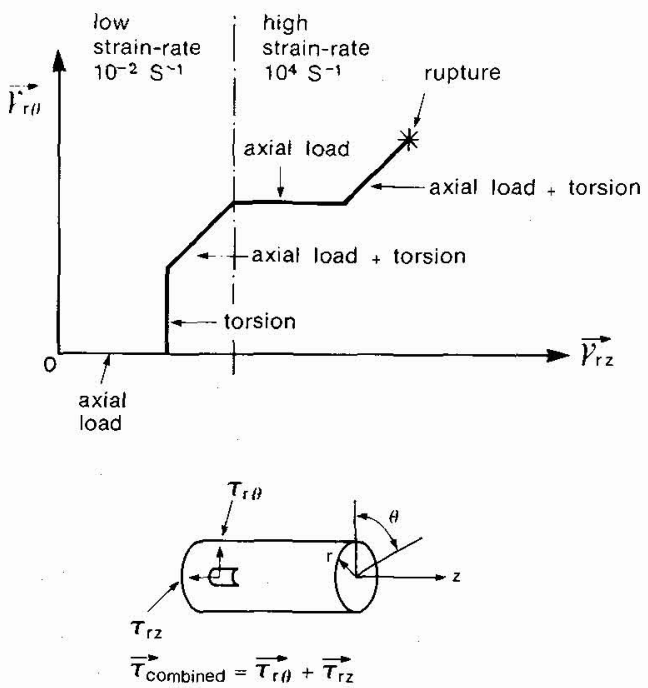

Fig.4: Example of complex strain and strain rate history.

\section{DESCRIPTION OF MEASUREMENT TECHNIQUES AND ANALYSIS}

The measurement of the stresses and strains acting on the specimen during dynamic testing are derived from records of strain gauges affixed to the bars as is usual in the standard strain-gauge technique. In the case of tests at low strain rate with no wave propagation, the double strain gauge stations I and II (Fig.2) permit to measure the axial and circumferential shear stresses acting on the gauge part. In the case of tests at high strain rate with wave propagation (Hopkinson bar), the double strain gauge stations I and II measure the incident-reflected and the transmitted parts of the axial and torsional pulses, respectively. In particular the double strain gauge station I measures the unloading wave of bar I (perfectly symmetric. of the incident wave on the specimen) and the wave reflected from the specimen. Because of the necessity of blocking the input bar very near to the specimen, in order to render negligible the delay between the arrival on the specimen of the axial and torsional waves, the resolution of incident and reflected waves is difficult.

The resolution of incident and reflected waves is also complicated by the cross-effects on the strain-gauge stations I and II of the axial and torsional waves. Therefore, in the Hopkinson bar case, the calculation of strains from the integration of the reflected pulses is very complex and might be affected by uncertainties. 
On the contrary, the transmitted axial and torsional waves are very clear (Fig.5) and can be used in the Hopkinson bar case to calculate the axial and circumferential shear stress on the gauge part of the specimens, after having been corrected from cross-effects and rephased in time due to the different speed of axial and torsional waves.

Due to the above-mentioned difficulties in recording and resolving the incident and reflected waves by strain-gauge measurements along the bar, a direct measurement of deformation on the specimen has been developed.

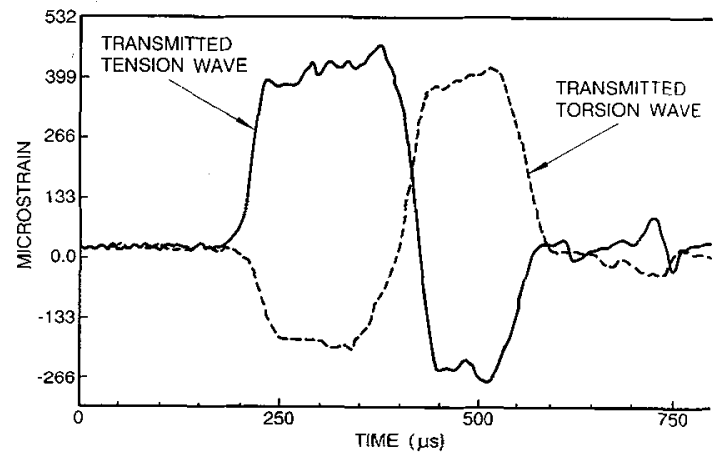

Fig.5: Transmitted axial and torsional waves.

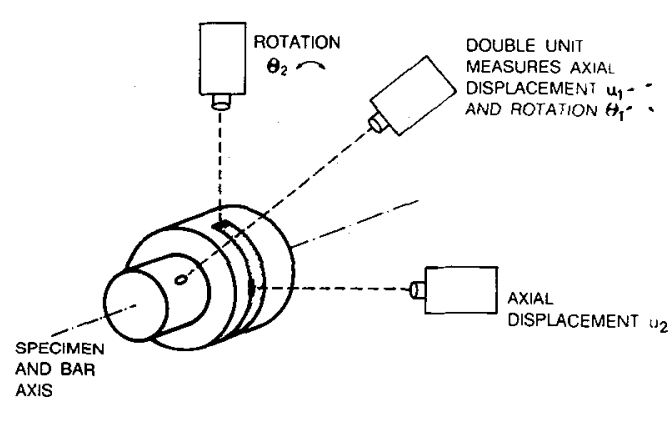

Fig.6: Displacement measurement set-up.

\subsection{Direct Measurement of Deformation}

In the case of quasi-static and medium strain rate testing, the determination of the shear strains in the specimen (both in the axial direction and in the circumferential direction) requires direct measurement of the relative axial displacement and relative angular displacement of the two cylindrical ends of the specimen. The short gauge length chosen for these biaxial tests results in very small displacements of the ends of the specimen and, therefore, a sensitive, high-resolution method of static and dynamic measurement of displacement - both linear and angular, is required. This has been achieved in a relatively simply way using a modified electro-optical displacement measuring device which is available commercially (cf. Zimmer A.G., U.D.T., Inc.). In simple terms the device is able to 'follow' the movement (up to high velocities) of a black/white edge (or a small white spot) and provides an electrical voltage proportional to the displacement of the edge. The displacement range is determined by the optical lens system used with the device and the resolution seems limited only by the internal noise generated in the phototube used to sense the black/white edge. The edges or targets which the device follows are produced on photographic paper and are fixed to the elastic cylindrical ends of the specimen.

In the particular application described here the device has served to record axial and rotational displacements of the two ends of the specimen of about 0.2 and $0.5 \mathrm{~mm}$ and $3^{\circ}$ and $8^{\circ}$, respectively, with resolutions of at least $1 \%$ (both statically and dynamically).

Simple wave theory in bars shows that the stress in an elastic pulse is proportional to the particle velocity. Since the electro-optical device measures the displacements of the cylindrical parts of the specimen which remain elastic, differentiation of the displacement-time record will give a stress-time record of the pulse transmitted through the gauge part, i.e. the specimen stress record. These records can be compared with the stress-time profile derived from the strain-gauge records, 
so that useful comparisons can be made not only for strain but also for the stress on the specimen. Fig.6 shows the disposition of the four devices used to measure displacement and rotation of the two ends of the specimen and Fig.7 shows typical displacement records.

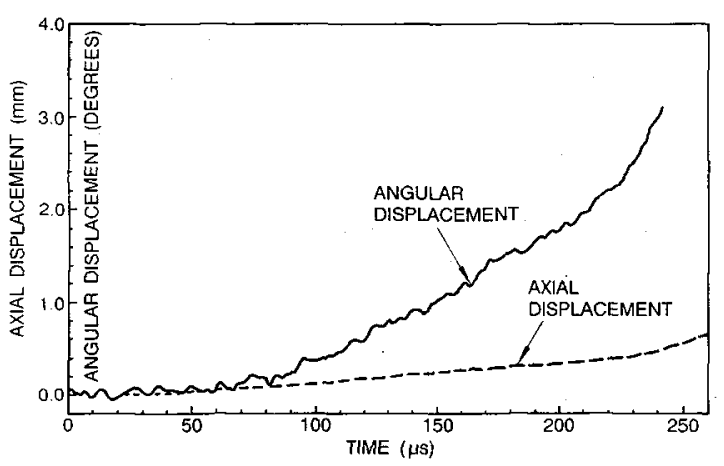

Fig.7: Angular and axial displacement records.

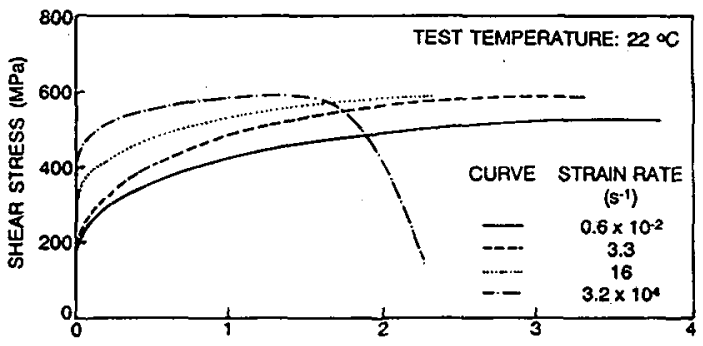

ENG. SHEAR STRAIN

Fig. 8: Engineering shear stressstrain curves of AISI 316

\section{RESULTS AND DISCUSSION}

The first tests have been performed with uniaxial loading conditions in order to check the performances of specimen, loading equipment and instrumentation under increasing testing difficulties.

The flow curves in uniaxial shear at low and medium strain rate for AISI 316 (Fig.8) show strain hardening up to strain values of about 2, after which there is saturation of flow stress (zero strain hardening); at high strain rate the saturation of flow stress is reached much earlier, at a strain of 1 . The reason for these differences of the shear flow curves at low to medium and very high strain rate is probably due to the presence of strain concentrations at a very high strain rate, as reported in /4/. Nevertheless, the shear flow curves of both materials at low, medium and high strain rate reach very high values of fracture strains and strainrates.

The equivalent flow curves obtained from the shear flow curves of Fig. 8 are rompared in Fig.9 with the true tension flow curves of the same AISI 316 which have been studied up to fracture following the Bridgman analysis /5/. From this comparison it follows that:

- The tension curves show strain hardening up to fracture, while the equivalent curves from the shear tests show saturation of flow stress.

- As a consequence of the above phenomenon, the true flow curve in tension and he equivalent flow curve in shear show a modest agreement at low strain, while it large strain they diverge, particularly at high strain rates.

- The fracture strain of the tension curves and of the equivalent curves from the shear tests are comparable.

- The above-mentioned observations are valid also taking into account that during necking the strain rate is about ten times higher than during uniform straining.

It follows that stress and strain characteristics depend on the deformation mode and that it is not possible to obtain a unique equivalent flow curve from tension and shear tests, as claimed in classical plasticity, because the physical phenomena are more complex than those included in the classical material models.

Successively the first tests have been performed on the 'bicchierino' specimen by combining axial and circumferential shear stresses.

The results are shown in Fig.10 where the axial and the circumferential shear stress-strain curves are reported together with their equivalent flow curve.

Also in biaxial shear the attained fracture strains are very large and the strain 
rates very high $\left(\sim 10^{4} \mathrm{~s}^{-1}\right)$. Refinements of the loading technique will permit strain rates up to $10^{5} \mathrm{~s}^{-1}$.

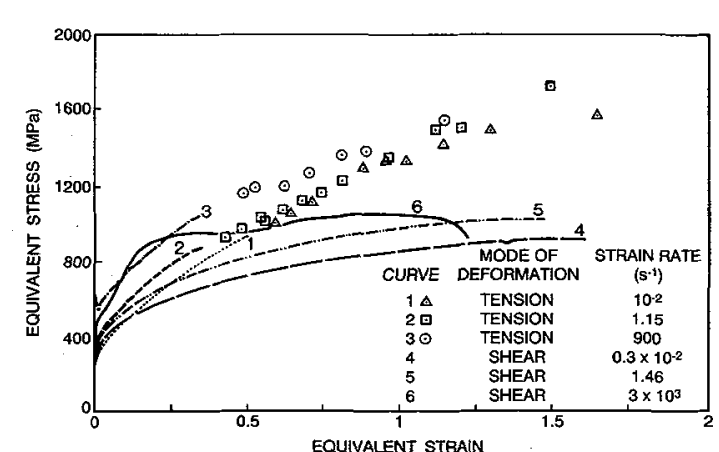

Fig.9: Uniaxial tests. Tension and equivalent stress-strain curves of AISI 316.

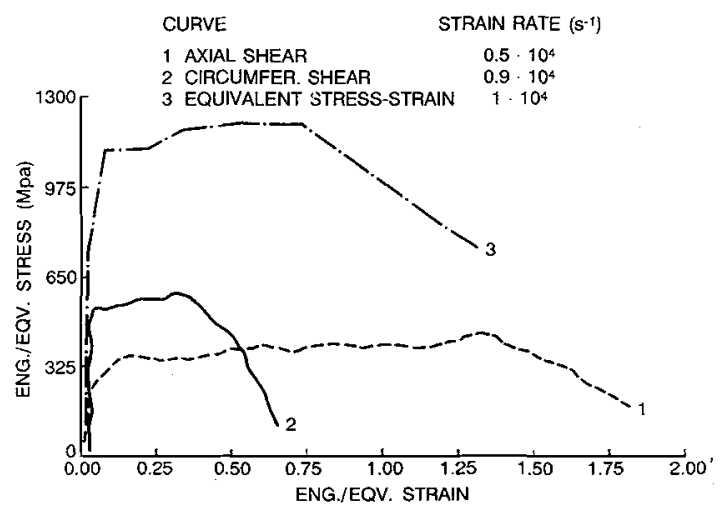

Fig.10: Biaxial shear test. Shear flow curves and equivalent flow curve.

\section{CONCLUSTONS}

It has been proved that a special cylindrical specimen having a thin $(0.25 \mathrm{~mm})$ ring-shaped gauge part permits to perform accurate biaxial shear experiments up to very large strains $(\sim 4)$ and to very high strain rates $\left(\sim 0.5 \times 10^{5} \mathrm{~s}^{-1}\right)$ which are characteristic of fast metal cutting and wire drawing, and of missile penetration. A special electro-optical technique has been developed in order to measure directly on the specimen the shear strains and the shear stresses along the axial and circumferential loading directions, permitting a check of the shear stress strain curves calculated from the complex wave propagation history along the tension-torsion Hopkinson bar. Equivalent stress-strain curves from shear tests of AISI 316 compared with tension curves showed discrepancies.

\section{REFERENCES}

1. Clifton, R.J., Dynamic Plasticity, Trans. ASME, J. Appl. Mech. 50 (1983) p.941-952.

2. Johnson, G.R., Hoegfeldt, M.M., Lindholm, U.S., Nagy, A.J., J. Eng. Mater. Technol. 105 (1983) 48.

3. Albertini, C., Montagnani, M., Ziczkowski, M., Laczek, S., Optimal design of a bicchierino specimen for double shear, Int. J. of Mechanical Sciences 32, 9 (1990) 729-741.

4. Albertini, C., Montagnani, M., Pizzinato, E.V., Rodis, A., Berlenghi, S., Gerini, G., Pazienza, G., Paluffi, A., Mechanical properties in shear at very high strain rate of AISI 316 stainless steel and of a pure iron, Proc. of the Int. Conf. on Shock-Wave and High Strain Rate Phenomena in Materials, University of California, San Diego (under publication by M. Dekker) (1990).

5. Bridgman, P.W., Studies in large plastic flow and fracture, MacGraw-Hill Company, New York (1952) 9-37. 